\title{
US duration and local trial spacing affect autoshaped responding
}

\author{
GARY A. LUCAS \\ Indiana University, Bloomington, Indiana \\ and \\ EDWARD A. WASSERMAN \\ University of Iowa, Iowa City, lowa
}

\begin{abstract}
The acquisition and maintenance of signal-directed pecking was examined in week-old Leghorn chicks responding to a keylight stimulus paired with heat. In contrast with previous studies using pigeons with food as the US, both speed of acquisition and asymptotic level of keypecking were a direct function of US duration. Experiment 2 examined responding using a within-subject design to isolate the effects of trial spacing on performance during the immediate trial from the effects on performance during a following trial of fixed length. These comparisons revealed a significant effect of intertrial interval (ITI), with less responding after shorter intervals. The effect of different temporal spacing was apparent in responding on the immediate trial, but not on the following trial. These local ITI effects were better predicted by a recent autoshaping model based on relative waiting time (Jenkins, Barnes, \& Barrera, 1981) than by a model based on relative US expectancy (Gibbons \& Balsam, 1981). However, neither model predicted the effect of US duration. A reexamination of the USduration literature suggested that the diversity of previous findings is consistent with the assumption that conditioned responding is an inverted U-shaped function of US duration.
\end{abstract}

When Brown and Jenkins (1968) described the tendency of pigeons to approach and contact a keylight stimulus predicting periodic food, they noted the similarity of the autoshaping procedure to standard Pavlovian conditioning arrangements. Subsequent research has pursued this similarity and has generally demonstrated an important role for the stimulus-reinforcer contingency in autoshaped responding (see Locurto, Terrace, \& Gibbon, 1981, for recent reviews). One Pavlovian variable that has not appeared to have much effect on autoshaped responding is the duration of the unconditioned stimulus (US) used in training (Balsam, Brownstein, \& Shull, 1978; Balsam \& Payne, 1979). The strength of responding is frequently reported to be a direct function of US magnitude in Pavlovian procedures when the amount or the intensity of the US is varied (Balsam \& Payne, 1979; Mackintosh, 1974). However, variations in US duration have produced equiv-

The authors wish to thank James Allison, Peter Balsam, and Elliot Hearst for their helpful comments on earlier versions of the manuscript. Requests for reprints should be addressed to Gary A. Lucas, Department of Psychology, Indiana University, Bloomington, Indiana 47405. This research was supported in part by Grants BNS 75-15905 and 79-14160 from the National Science Foundation. During the study, the first author was supported by a Neurobehavioral Sciences postdoctoral fellowship through NIMH Training Grant 1T32MH15172. ocal findings across a variety of paradigms (e.g., Church, LoLordo, Overmier, Solomon, \& Turner, 1966; Frey \& Butler, 1973; Overmier, 1966; Riess \& Farrar, 1973; Runquist \& Spence, 1959; Wegner \& Zeaman, 1958). A review of these findings led Balsam and Payne (1979) to conclude that US duration may not produce effects in Pavlovian conditioning that are comparable to those found for other manipulations of US magnitude.

The nature of the US-duration effect is important for two recent models of autoshaping performance. One model, proposed by Gibbon and Balsam (1981), follows from Gibbon's scalar expectancy theory (Gibbon, 1977) and is based on a relative expectancy hypothesis. This model predicts autoshaped responding based on the ratio of US expectancy during the CS to the expectancy of the US across the entire background cycle. Because the same US magnitude is "expected" for both trial and background conditions, relative expectancy is not affected by US duration. Thus, the relative expectancy hypothesis predicts no change in autoshaped performance for different US durations. The second model of autoshaped performance is based on a similar temporal relationship between trial and background expectancies, expressed in terms of relative waiting time (Jenkins, Barnes, and Barrera, 1981). The predictions of the relative waiting time hypothesis are de- 
scribed by the authors as virtually identical to those of the relative expectancy hypothesis. The relative waiting time hypothesis does not directly address the effect of US duration. However, in discussing the differences between the two models, neither Jenkins et al. (1981) nor Gibbon (1981) suggests that any differential effect of US duration is predicted between the two models.

The predictions of the relative waiting time hypothesis so overlap those of the relative expectancy hypothesis that at least one author (Gibbon, 1981) has expressed doubt that differential predictions can be obtained from the two approaches. However, at least conceptually, a distinction between the two models can be made regarding the direction in which timing information is used. For the relative waiting time model, the critical value is the time spent "waiting" since the last US. For the relative expectancy model, the critical time is the time "expected to wait" until the next US. A potential distinction between these two orientations may be possible if the source of the timing information used for changing an expectancy can be isolated.

The present studies are addressed to the predictions of these recent autoshaping models. In particular, they challenge the interpretations of Balsam and Payne (1979) and of Gibbon and Balsam (1981) that exclude a US duration effect for autoshaped responding. Included in this study is a detailed examination of the within-session effects of trial spacing on autoshaped responding. These data, reported in Experiment 2, appear to distinguish between the relative expectancy model and the relative waiting time model, when these models are extended to predict local changes in responding.

\section{EXPERIMENT 1}

The present study used a thermal reinforcer to examine the effects of US magnitude on autoshaped keypecking. Previous findings with the heat-reinforcement procedure have shown many parallels to the food-reinforcement procedure used with pigeons, for example, the effects of a response-omission contingency (Wasserman, 1973), random stimulusreinforcer pairings (Wasserman, Hunter, Gutowski, \& Bader, 1975), partial reinforcement (Wasserman, Deich, Hunter, \& Nagamatsu, 1977; Wasserman et al., 1975), and second-order conditioning (Deich, 1982). Here, the comparison was extended to US duration.

\section{Method}

Subjects. The subjects were 56 experimentally naive White Leghorn cockerels obtained from a commercial hatchery (Welp Inc., Bancroft, Iowa) on their 2nd day after hatching. On arrival in the laboratory, the chicks were transferred to a commercial brooder that maintained a temperature range of $29^{\circ}$ to $35^{\circ} \mathrm{C}$, depending on location in the brooder. Commercial chick starter (Purina Startena) and water were freely available. At least $16 \mathrm{~h}$ before the beginning of experimental sessions, chicks were isolated in stainless steel cages $(18 \times 24 \times 18 \mathrm{~cm})$ in a constantly lighted room maintained between $25^{\circ}$ and $27^{\circ} \mathrm{C}$. Six chicks that appeared weak or failed to eat readily after $16 \mathrm{~h}$ of isolation were replaced by subjects run the following week. The experiment was run in two replications counterbalanced across hatches.

Apparatus. Four identical conditioning chambers were used. Each aluminum chamber floor measured $18 \times 18 \mathrm{~cm}$ and was covered by a disposable sheet of white paper. The sides of the chamber were formed by three 29.5 -cm-tall aluminum walls and a fourth wall, made from $1.3-\mathrm{cm}$ hardware cloth, to facilitate airflow. On the wall opposite to the hardware cloth, a response key was recessed $2.5 \mathrm{~mm}$ behind a circular opening, $1.9 \mathrm{~cm}$ in diameter, and centered $10 \mathrm{~cm}$ above the chamber floor. $\mathbf{A}$ minimum force of $.04 \pm .01 \mathrm{~N}$ through a distance of $2.5 \mathrm{~mm}$ was necessary to operate the magnetic reed switch that transduced the keypeck. The translucent Plexiglas key was transilluminated through an orange filter by a No. 44 bulb in a miniature display projector (Industrial Electronics Equipment, series 10) operated at $5.5 \mathrm{~V} \mathrm{dc}$.

Each experimental chamber was situated in an individual refrigerated enclosure $(81 \times 44 \times 74 \mathrm{~cm})$. Air temperature within the chamber was maintained at $5^{\circ} \pm 3^{\circ} \mathrm{C}$. The chamber was located approximately $12 \mathrm{~cm}$ left of the refrigerated enclosure's center (across the long dimension) on a wooden floor raised $22 \mathrm{~cm}$ above the base of the enclosure. Two $15-\mathrm{W} 120-\mathrm{V}$ ac bulbs were mounted symmetrically on the same plane as the front wall, $18 \mathrm{~cm}$ above the top of the chamber and $5 \mathrm{~cm}$ lateral to the aluminum sidewalls. These lights remained on continuously during the session and provided general illumination for the chamber.

The unconditioned stimulus (US) was a combination of visual and infrared illumination provided by a Sylvania $250-\mathrm{W} 120 \mathrm{~V}$ ac frosted reflector heat lamp centered $30 \mathrm{~cm}$ above the chamber floor. Temperature measures at the level of the floor indicated that the intensity of infrared illumination varied less than $7 \%$ from the center of the chamber to the corners.

The refrigerated enclosures were sound attenuated. In addition, white noise was continuously sounded in the room housing the enclosures. Experimental scheduling and recording were provided by a PDP 8/A minicomputer (Digital Equipment Corporation) using the SKED software system (Snapper, Stephens, \& Lee, 1974).

Procedure. Experimental training began on the 7 th day after hatching. The chicks were randomly assigned to one of seven experimental groups $(\mathrm{N}=8)$ that varied according to the duration of the heat-lamp operation $(.25, .5,1,2,4,8$, or $16 \mathrm{sec})$.

In Phase 1, for each group, the conditioned stimulus (CS) was a 6-sec illumination of the orange keylight, followed immediately by operation of the heat lamp for the specified duration. A variable ITI averaging $36.6 \mathrm{sec}$ was timed from the offset of the heat lamp to the onset of the next CS. Details regarding the distribution of the ITI are described in Experiment 2. Each session was terminated after 40 daily trials.

Phase 1 training continued for 4 days. Individual food and water supplies were replenished in the home cage following each experimental session. Access to food was available for the next 12 to $16 \mathrm{~h}$, but food was withheld for at least $8 \mathrm{~h}$ prior to the next daily session to ensure moderate levels of activity at the time of training.

In Phase 2, to assess the associative effects of US duration, on the following day each subject was treated as in Phase 1, except that a fixed 2-sec heat-lamp duration was presented to all subjects. It was expected that, if the effects of US duration were associative, then differences in US duration should influence the strength of conditioning to the CS. Any such differences in associative strength would then be apparent in a transfer condition in which US duration was held constant. However, if the obtained differences in performance in Phase 1 were produced by 
nonassociative effects of motive or arousal specific to the immediate presentation of different US durations, then no differences in performance would arise in Phase 2, since the source of arousal, US duration, remained constant across groups.

\section{Results}

In Phase 1, subjects trained with longer US durations acquired keypecking sooner than did subjects trained with shorter durations. The mean number of trials presented before a subject first responded to the CS is shown for different reinforcer durations in Figure 1. Note that US duration is plotted on a logarithmic scale. The rate of acquisition was well described as a negative power function of reinforcer duration. The product-moment correlation between the log mean US duration and log mean trial to first peck was -.983 . Analysis of variance confirmed a significant effect for rate of acquisition $[F(6,44)=$ $5.87, \mathrm{p}<.001]$. Data for three subjects in the .25 -sec group and for two subjects in the .5-sec group that failed to acquire keypecking were excluded from this analysis and the above correlation, but were included in the percentage of trials-with-a-peckmeasure used below. ${ }^{1}$

The effect of US duration on asymptotic responding was examined using the percentage of CS trials on which at least one keypeck occurred. As found for rate of acquisition, the percentage of trials with a peck was a direct function of US duration, and responding appeared stable by the 3rd day of train-

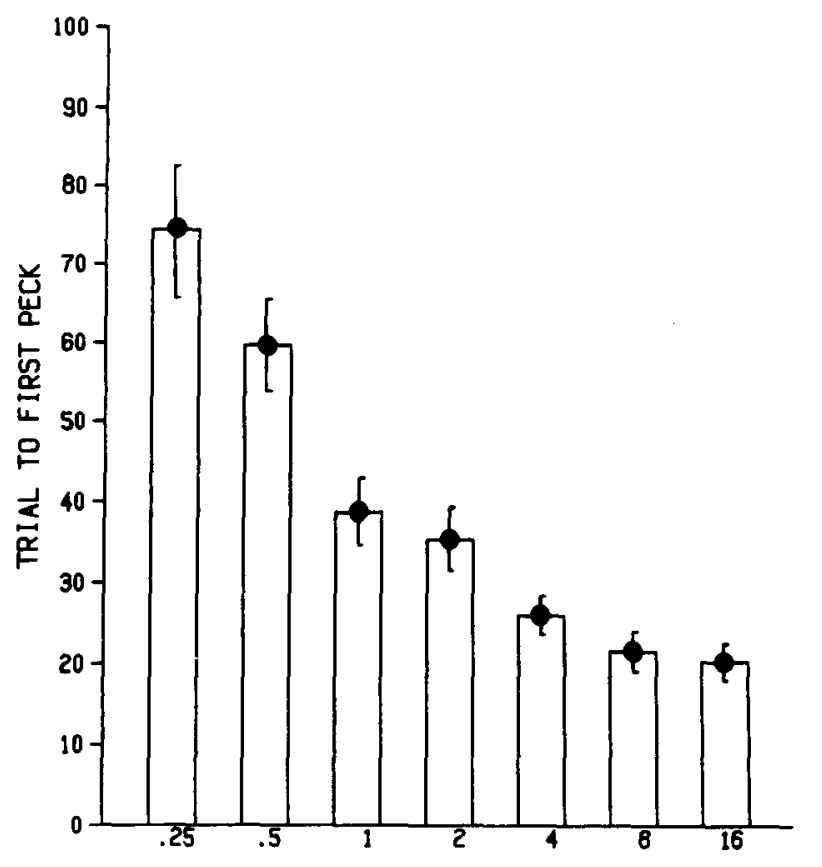

HEATLAMP DURATION ( SEC )

Figure 1. The mean number of CS trials presented before the first trial with a peck as a function of US duration. Brackets indicate standard errors.

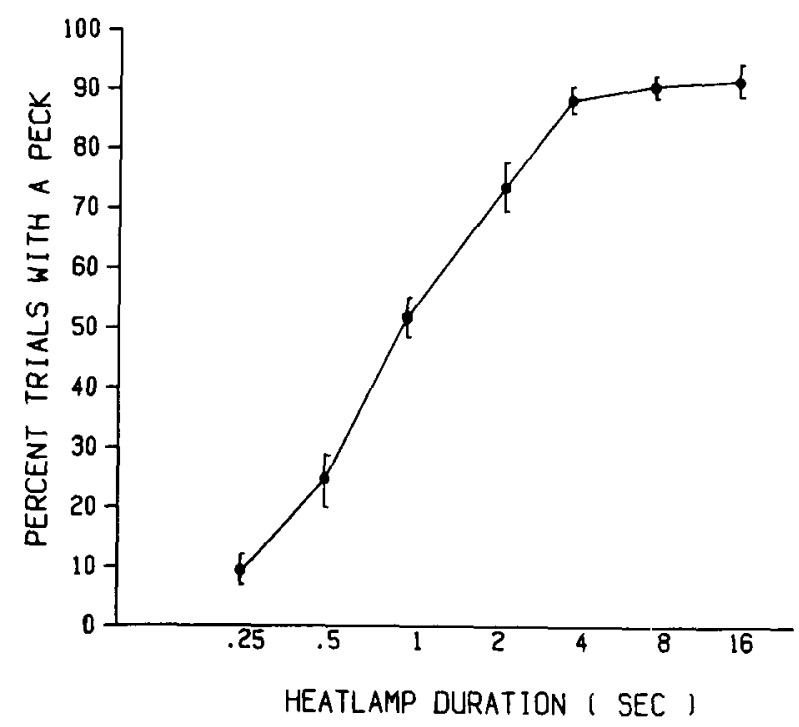

Figure 2. The mean percentage of CS trials with a peck for each US duration group. Data are averaged across Days 3 and 4. Brackets indicate standard errors.

ing. Analysis of variance on this measure across Days 3 and 4 supported these conclusions, indicating a highly significant effect of US duration $[F(6,49)=$ $32.99, \mathrm{p}<.001]$. The mean percentage of CS trials with a peck combined across Days 3 and 4 is presented in Figure 2. The comparison across days revealed a very stable percentage of trials with a peck, averaging $61.3 \%$ and $60.3 \%$ for Days 3 and 4 , respectively. Neither this difference $[F(1,49)<1.0]$ nor the interaction between US duration and day of training $[F(1,49)<1.0]$ was significant. Previous work with this paradigm (Wasserman, Deich, et al., 1977; Wasserman, Hunter, et al., 1975) has demonstrated rapid learning to stable response levels in 3 to 5 days of training. Inspection of individual group curves also supported the conclusion that response measures for all groups were near asymptotic levels.

A similar analysis across Days 3 and 4 using the mean latency to respond on trials with a peck, or the mean response rate during the $\mathrm{CS}$, supported the above findings. For both these measures, the analysis excluded the five subjects that failed to keypeck. As shown in Table 1, the latency to respond was shorter and response rate was higher for those groups trained with longer heat-lamp durations. For both measures, a significant effect was found for US duration, $F(6,44)=5.63, p<.001$, for the latency measure and $F(6,44)=5.90, p<.001$, for the response rate measure. Again, the comparisons across days and the US duration $x$ day interaction were not significant.

Although each group received the same US duration $(2 \mathrm{sec})$ in Phase 2, the percentage of trials with a keypeck continued to vary as a direct function of the US duration used in training. Mean scores for 
Table 1

Mean Latency (in Seconds) to Respond to the CS on Trials With a Peck and the Mean Rate of Responding During the CS as a Function of Heat-Lamp Duration

\begin{tabular}{cccccc}
$\begin{array}{c}\text { Reinforcer } \\
\text { Duration* }\end{array}$ & N & \multicolumn{2}{c}{ Latency } & \multicolumn{2}{c}{ Responses per Minute } \\
\cline { 1 - 4 } .25 & 5 & 2.89 & .31 & 5.2 & 1.9 \\
.50 & 6 & 2.28 & .19 & 13.6 & 2.9 \\
1.00 & 8 & 2.37 & .23 & 41.4 & 11.4 \\
2.00 & 8 & 1.93 & .13 & 50.9 & 8.5 \\
4.00 & 8 & 1.71 & .16 & 67.8 & 14.0 \\
8.00 & 8 & 1.79 & .20 & 89.1 & 14.6 \\
16.00 & 8 & 1.59 & .18 & 77.4 & 17.9 \\
\hline
\end{tabular}

Note $\rightarrow$ Data are means for performance on Days 3 and 4 for each group. $N=$ number of subjects responding. *In seconds.

Table 2

Mean Percentage of Trials With a Peck on Day 5, Using a 2-Sec Heat Lamp, as a Function of the Duration of Heat Used in Phase 1 Training

\begin{tabular}{lrrrrrrr}
\hline & \multicolumn{7}{c}{ Reinforcer Duration (in Seconds) } \\
\cline { 2 - 8 } & \multicolumn{1}{c}{25} & \multicolumn{1}{c}{.5} & 1.0 & 2.0 & 4.0 & 8.0 & 16.0 \\
\hline Mean & 25.0 & 35.6 & 58.8 & 64.1 & 73.4 & 67.5 & 82.5 \\
SE & 7.0 & 8.6 & 8.4 & 7.9 & 6.4 & 8.6 & 6.5 \\
\hline
\end{tabular}

Note-Data are group means $(N=8)$.

the percentage of trials with a peck on Day 5 are shown in Table 2. Analysis of variance on individual scores indicated a significant effect of the previous reinforcer duration $[F(6,49)=7.20, p<.001]$. This finding strongly supports the conclusion that the effects of US duration on autoshaped keypecking were associative, and not merely an artifact of differences in motive or arousal resulting from the immediate presentation of a specific US duration.

\section{Discussion}

Balsam and Payne (1979) examined the effects of a wide range of feeder durations on autoshaped responding in pigeons. Not having found any effect of feeder duration on keypecking, and having reviewed a number of Pavlovian studies of US duration that failed to find a distinct effect, they suggested that perhaps US duration does not produce the same behavioral effects as US intensity, quality, or numerosity. More specifically, they argued that the vigor of the CR might depend more on US onset than on US duration.

Although there is evidence that long intermittent US presentations support more responding than continuous presentations in the same time period (Furedy, 1967; Overmier, 1968), the robust effect of US duration obtained with the current heat-reinforcement procedure clearly demonstrates that US duration can also produce strong effects on conditioned responding comparable to those obtained with other mea- sures of US magnitude (see Balsam \& Payne, 1979, and Mackintosh, 1974, for recent summaries).

It might be argued that the extended duration of the heat-lamp operation could result in a subjective difference in stimulus intensity. That is, longer durations of heat may sum to produce a difference in perceived US intensity that is qualitatively distinct from intensity differences produced by changes in feeder duration. Although we agree that temporal summation may play an important role in mediating differences in US duration, the assumption of qualitative differences in summation for different reinforcers is often post hoc and does not appear to explain the varied effects of US duration reported in the literature. For example, although the comparison between heat duration and feeder duration might suggest a qualitative difference, it is not clear why the effects of heat duration should differ from those of shock duration (Mowrer \& Solomon, 1954; Salz, Kitai, \& Asdourian, 1963; Wegner \& Zeaman, 1958) or the duration of cool air (Furedy, 1967).

Variations in US duration introduce an additional complexity through the possibility that the duration of the US may alter the relative temporal relationship between trials and background stimuli. Previous work (see Gibbon \& Balsam, 1981) has shown that acquisition and maintenance of autoshaped responding are directly related to the ratio of trial to overall background durations. However, Balsam and Payne (1979) found no evidence to argue that US duration should be included in calculating the overall cycle duration. Instead, they concluded that the pigeon's autoshaped performance was directly related to ITI excluding reinforcer duration. In the present study, a substantial increase in response measures was obtained between US durations of, 25 and $1 \mathrm{sec}$. It is difficult to argue that these changes in behavior were produced by changes in relative trial spacing when the addition of reinforcer time to the total trial cycle only amounted to a mean change of from 42.85 to $43.60 \mathrm{sec}$ in the overall trial cycle. Had performance changes required substantially longer changes in US duration, then a possible contribution of US duration to relative trial spacing might have been implicated. However, the substantial increase in responding reported here, even across a .75 -sec increase in US duration, cannot be explained by changes in trial spacing.

It is also possible that the amount of heat engendered by the operation of the heat lamp at very brief durations may be near the chick's threshold of perception. In this case, the effects of shorter durations might be confounded by a perceived intermittency of the reinforcer. Furthermore, this effect might be potentiated if the intensity of the heat lamp varied substantially across areas of the chamber so that perception of the heat lamp would 
vary according to location. These explanations seem unlikely in the present design, for two reasons. First, measures of temperature change across the chamber indicated that more than $93 \%$ of the infrared illumination reaching center floor was present within $1 \mathrm{~cm}$ from the corner of the chamber floor. Second, although the question of intermittent perception cannot entirely be ruled out at the very briefest durations, this argument does not seem adequate to account for the main effect of US duration across the range of values examined here. It would be necessary to postulate a rather extreme variance in perceptual thresholds to account for differences in performance across longer US durations by a similar mechanism.

\section{EXPERIMENT 2}

Previous studies have shown that trial spacing is directly related to the rate of acquisition and the level of responding maintained in autoshaping (see Balsam \& Payne, 1979). Recently, the effects of trial spacing have gained increasing theoretical attention in models emphasizing the relative waiting time between US events (Jenkins et al., 1981) or relative US expectancy (Gibbon, 1981; Gibbon \& Balsam, 1981). The distinction between the relative waiting time model and the relative expectancy model revolves around the question of how time is incorporated into the excitatory strength of a stimulus condition. For the relative expectancy model, excitatory strength is a function of expectancy, that is, some forwardlooking estimate of mean time to the next US. For the relative waiting time model, the excitatory strength of a stimulus depends on the average time spent waiting for the US in that condition. Thus, in the waiting time model, excitatory strength depends on an estimate of the mean delay since the last US.

Although a conceptual difference exists between the relative expectancy hypothesis, with its emphasis on predicting the next US, and the relative waiting time hypothesis, with its emphasis on assessing the delay since the last US, an experimental distinction is difficult to define (see Gibbon, 1981). In the usual autoshaping procedure, both models make identical predictions when mean trial spacing is examined. However, it seemed to us that a distinction might be found regarding how each model could accommodate a change in US frequency. Essentially, we asked how quickly each model would adjust for variations in trial spacing.

To answer this question, we assumed that, in order to adjust to a new intertrial spacing, it would be necessary for recent changes in timing to affect the average estimate of.the intertrial interval. As Gibbon and Balsam (1981) note, such an adjustment would be required for an expectancy to undergo extinction. The implication of this assumption is that the aver- age expectancy or waiting time must be based on some moving average of the more recent intertrial intervals. This suggests that a local change in interreinforcer timing should produce a measurable effect on the average estimate of trial spacing. An experiment by Jenkins et al. (1981) appears to support this assumption by showing an immediate change in the rate of acquisition for autoshaped pecking following a single long interfood wait.

Recently, Killeen (1981) has proposed a model for an exponentially weighted moving average (EWMA) to account for local averaging effects. Such a mechanism would seem to be essential to adjust for changes in trial spacing. However, we should point out that both the relative expectancy model and the waiting time model were formulated on average expectancies (or waits) and that neither model explicitly states how such averages are adjusted. Thus, while we feel that the question of local trial spacing is ultimately important to each model, it should be clear that we are testing an extension of these models to a problem that they have not explicitly addressed.

In order to examine the local effects of trial spacing, the ITI was structured such that a range of variable ITIs alternated with a constant interval $(36 \mathrm{sec})$, which approximated the arithmetic mean of the distribution $(36.6 \mathrm{sec})$. This procedure served to hold the effects of the interval on trial $n-1$ constant, and thus helped to isolate the local effects of the specific interval on trial $n$. Note that the CS on trial $n$ may be considered to sample performance after different "waiting times." Data in the constant ITI components, those presented on alternate trials, were recorded separately for each variable ITI so that the effect of the interval length could also be assessed on responding in the following trial. Performance on the following trial was expected to reflect any local change in the average "expectancy" of the US that may have resulted from the length of the ITI on trial $n$.

\footnotetext{
Method

The design of Experiment 2 was embedded in the procedure for scheduling the ITIs in Experiment 1. Thus, the data reported herein were collected from the subjects in Experiment 1 concurrently with the running of that procedure.

The distribution of the ITIs was arranged such that one of five ITls $(6,12,24,48$, or $96 \mathrm{sec})$ alternated with a 36 -sec ITI, the approximate mean of the entire distribution. Each of the five variable ITIs (except the first of the session) was preceded by the mean (36-sec) ITI. Data were recorded in separate categories following each of the variable ITI lengths, and data for the 36-sec ITI were grouped according to the duration of the previous ITI. The five variable ITIs were scheduled using a sample-withoutreplacement procedure so that each of the five intervals was selected twice every 10 opportunities. (Opportunities for the variable ITI alternated with the mean ITI.) Repeating this procedure twice per session produced a total of 40 daily trials, 20 varying around the mean and 2036 - $\mathrm{sec}$ values, equally represented in the first and second half of each session.
} 
Results

The local effects of the ITI were examined using the percentage of trials with a peck for individual subjects averaged across Days 3 and 4 . Data for the "mean" (36-sec) ITI components were treated separately. Averaged performance across the five "variable" ITI components is shown in Figure 3. Between the 6-sec and 48-sec ITIs, the probability of responding was a direct function of the interval since the last trial. Analysis of variance on these data revealed a significant effect of ITI $[F(4,196)=2.53, p<.05]$, as well as the previously reported effect of US duration $[F(6,49)=27.14, p<.001]$. There was no significant interaction between US duration and the length of the interval $[\mathrm{F}(24,196)<1.0]$. Individual planned comparisons at the .05 level indicated that performance after the 6-sec ITI was significantly lower than performance after the 24-sec and 48-sec intervals and that performance after the 12-sec interval was significantly lower than performance after the 48-sec interval. Performance after the 96-sec interval did not differ significantly from the other intervals.

Analysis of the data from the 36-sec ITI trials using the trial-with-a-peck measure revealed no significant effect of trial $\mathbf{n}$ ITI on responding in trial $\mathbf{n}+1$ $[F(4,196)<1.0]$. Again, the effect of US duration was significant $[F(6,49)=35.98, p<.001]$, and no US duration $x$ intertrial interval interaction was evident $[F(24,196)<1.0]$. The combined mean for the percentage of trials with a peck after the $36-\mathrm{sec}$ interval is presented in its respective position in Figure 3.

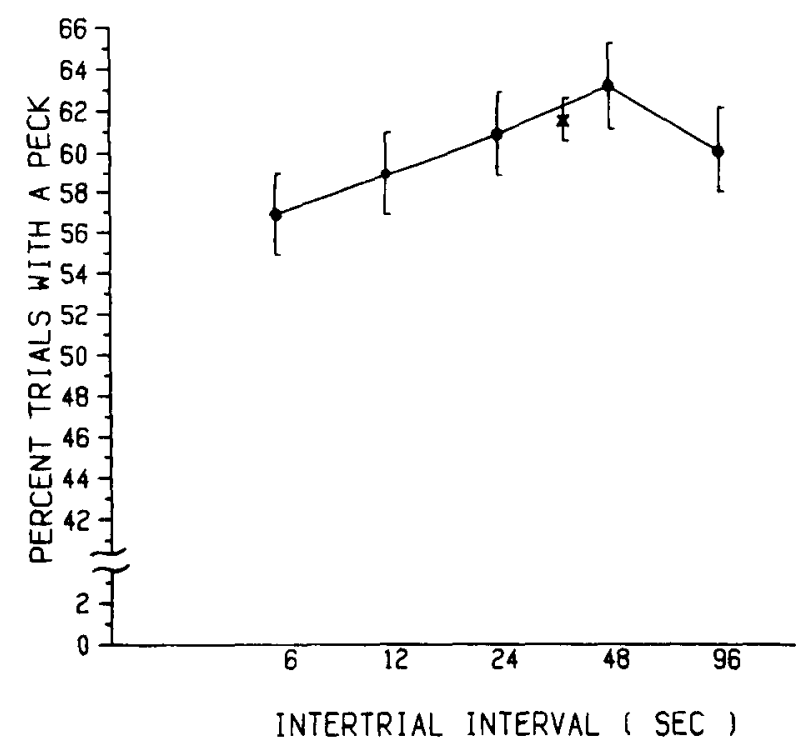

Figure 3. The mean percentage of CS trials with a peck as a function of the length of the interval preceding the trial. Data are combined scores for Days 3 and 4 averaged across all US duration groups. The $x$ indicates the average for the "mean" 36-sec interval presented on alternate trials. Brackets indicate standard errors.

\section{Discussion}

Following a number of inventive tests for the effects of ITI duration, Jenkins et al. (1981) concluded that the slower acquisition that was obtained in autoshaping procedures with shorter interfood intervals could not be explained on the basis of local interference effects. The present evidence for an effect of ITI duration within sessions is also not readily explained by an interference account. If the memory of the previous trial interferes with performance on the subsequent trial, then stronger interference effects should be produced by arranging more salient events on the previous trial. One of the most salient features of the trial in the present procedure was the operation of the heat lamp. An interference account should then predict stronger interference effects for subjects run with longer heat-lamp durations. However, there was no significant interaction of heat-lamp duration with ITI. The absence of an interaction across a broad range of US durations would seem to weaken the interference interpretation.

The effects of trial spacing found here represent small shifts in response tendency on a trial-by-trial basis. Although the within-session effects of ITI were relatively small, the direction of change was consistent with the more robust effects of trial spacing reported for between-subject comparisons (see Balsam \& Payne, 1979). In the absence of any obvious alternative, it seems most parsimonious to assume that the local effect of ITI length depends on small variations in the same momentary expectation or waiting time estimate assumed to be an important determinant of response tendency when averaged across longer time periods (Gibbon, 1981; Gibbon \& Balsam, 1981; Jenkins et al., 1981). The assumption is that recent variations in ITI may have a small, but measurable, effect on the subject's momentary estimate of these averages (cf. Killeen, 1981).

If these assumptions are allowed, then the betweentrial comparisons reported here appear to provide a way of distinguishing between the relative waiting time hypothesis (Jenkins et al., 1981) and the relative expectancy hypothesis (Gibbon, 1981; Gibbon \& Balsam, 1981). The distinction turns on whether, at the onset of trial $n$, the time since the last US should be included in the average expectancy or waiting time. Consider the occasion when the intertrial interval on trial $\mathrm{n}$ is very short compared with the previous average. If the new average is based on the time spent waiting, then the current waiting time is always known when the CS is presented on trial $n$ and must be included in the local average. However, if the performance on trial $\mathrm{n}$ is a function of some US expectancy measure, then the expectancy during trial $n$ cannot change until the interreinforcer interval on trial $n$ exceeds the previous average, or until the US is presented early. In effect, expectancies are up- 
dated only when a US, or its omission, occurs (see Gibbon \& Galsam, 1981, p. 247). The absence of the US at shorter-than-average intervals is part of the average expectancy. Alternately, the expectancy function should exercise a strong influence on performance in the following $(n+1)$ trial, since the interval on trial $n$ provides the most recent sample of interreinforcer time.

The present data support the waiting time hypothesis by showing a significant effect of ITI on trial $\mathrm{n}$ but no significant effect on the following trial. That is, the amount of time that passed since the last US presentation influenced the amount of responding directed to the CS, but the length of the previous interreinforcer interval did not significantly alter responding to the CS. These results suggest that the time spent waiting since the last US is the better predictor of responding within the autoshaping session.

Although these results suggest that local changes in US frequency affect autoshaped performance, Jenkins et al. (1981) appear to have reached a different conclusion. Their studies did not reveal a significant difference in the pigeon's keypecking, despite differences between groups in local interfood timing prior to CS-US pairings. One possible reason for this difference may be simply that the large number of subjects and the within-subject design employed here provided a more sensitive test of the local spacing effect. Indeed, even in our results the difference in performance following a local change in trial spacing was relatively small, suggesting that a recent change in trial spacing is given only a small weighting in the overall average.

In support of this argument, we note that several of the studies reported by Jenkins et al. (1981) demonstrate trends in performance that are consistent with our results. For example, in their Experiment 2 (1981, pp. 257-259), differences in local inter-US spacing were examined by presenting unsignaled food prior to a CS-US pairing while the average inter-US interval was held constant at $60 \mathrm{sec}$. When the interval since the last US was $300 \mathrm{sec}$ (Group After), the authors reported a median of 16 trials to acquisition of pecking. However, the median number of trials to acquisition was increased to 24 trials when two 30 -sec interfood intervals preceded the test trial (Group Before-After) and to 28 trials when four 30 -sec interfood intervals preceded the test trial (Group Before). Although these differences were apparently not significant, a clear trend was suggested by the quicker acquisition following longer interfood intervals.

A similar effect was reported in their Experiment 9 (Jenkins et al., 1981, pp. 267-268). In this study, CS-US pairings were interposed in a fixed series of unsignaled US presentations. The onset time for the CS was varied between 3 and $55 \mathrm{sec}$ following a midtrial US across five groups. Here, the authors reported a suggestive trend (again nonsignificant) with higher rates of responding after longer interfood intervals. These observations suggest that the local effects of trial spacing reported in the present study are probably not limited to the heat-reinforcement paradigm.

\section{GENERAL DISCUSSION}

While our Experiment 2 asserts a distinction between the relative expectancy hypothesis (Gibbon \& Balsam, 1981) and the relative waiting time hypothesis (Jenkins et al., 1981), it should be clear that the molar predictions of these models are virtually identical. The distinction posed here addresses the question of when the passage of time alters trial-by-trial performance. As such, our procedure may be considered to test an extension of these models to the problem of local changes in trial spacing. The results of Experiment 2 suggest that time spent waiting since the last US affects performance during the immediate CS, even before a change in US frequency could be determined. This finding favors the waiting time interpretation. However, a more pressing problem for these models is the robust effect of US duration reported in Experiment 1, which is not predicted by either the waiting time or the expectancy model.

\section{A US-Duration Function}

Balsam and Payne (1979) suggested that US duration may not produce a consistent effect in classical conditioning. This conclusion was based, in part, on the failure of feeder-duration manipulations to significantly alter the pigeon's autoshaped keypecking. In addition, they cited a number of studies, primarily those using heart rate and GSR measures, that failed to report an effect of US duration (Bitterman, Reed, \& Krauskopf, 1952; Coppock \& Chambers, 1959; Furedy, 1967; Mowrer \& Solomon, 1954; Overmier, 1966; Wegner \& Zeaman, 1958).

The US duration function reported in Experiment 1 appears to conflict with the above literature. However, reexamination of the literature suggests an alternative interpretation. First, note that in several of these studies the number of different US durations examined was relatively small, and thus not a very powerful test of the US duration function. And in the Overmier (1966) study, although no difference was found in heart rate conditioning, the CS associated with the longer shock duration supported stronger avoidance responding in subsequent tests. In addition, several studies have reported a significant effect of US duration, although the direction of change has not always been consis- 
tent. These include descriptions of a bitonic relation between conditioned responding and US duration (Church et al., 1966; Frey \& Butler, 1973) or superior conditioning with a shorter US duration Runquist \& Spence, 1959; Salz et al., 1963). Joining the latter is a recent autoshaping study using rats (Allison, Note 1).

Any conclusion about the shape of the US duration function may also depend on the range of durations studied and type of response measure used (e.g., Balsam et al., 1978; Overmier, 1966; Riess \& Farrar, 1973). In this regard, it seems worth noting that the present study would have revealed no clear effect of US duration had it examined only those durations between 4 and $16 \mathrm{sec}$, a fairly respectable range. And the apparent downturn in response rate between the 8-sec and 16-sec heat-lamp durations (see Table 1) suggests that a different outcome might have been found if somewhat longer US durations had been examined. Recent work (Lucas \& Deich, Note 2) using the heat-reinforcement paradigm has confirmed that a downturn in performance may occur at longer durations.

The data presented by Church et al. (1966) provide strong support for an inverted U-shaped function for US duration. This finding was based on data obtained across a wide range of shock durations. However, the effect of shock duration also varied with intensity. Depending on the intensity and the durations examined, the Church et al. data could be used to support the conclusion that a longer US duration produced larger CRs or smaller CRs, or had no effect. Taken together, these observations, and the variety of findings cited above, seem most compatible with the conclusion that conditioned responding often follows an inverted U-shaped function of US duration. However, the convexity of the function appears to interact with other motivational parameters (Church et al., 1966).

\section{The Feeder-Duration Problem}

One still unanswered question is why no evidence has been obtained for an effect of US duration following changes in feeder duration (Balsam \& Payne, 1979). This point is probably best answered by comparing the heat-reinforcement procedure used here with the feeder-duration procedure. When the duration of the heat-lamp operation supports rapid conditioning, the warming chicks exhibit a characteristic action pattern that includes a pronounced leg flexion and wing extension (Wasserman, 1973). However, when the quality of the US is poor, the response is a minimal movement of the wing with no clear extension or leg flexion.

In the feeder procedure, the response to a potent US is a rapid orientation to the food hopper followed by an approach to the grain, pecking, mandibulation, and swallowing. As in the heat-lamp procedure, the US arouses a distinct action pattern.
However, in the feeder procedure, the complete US is composed of the serial presentation of a conditioned signal, the sound and light of the grain delivery, and the orientation and approach behaviors that bring the pigeon in contact with the grain to complete the sequence. The approach sequence is required to complete the compound. When the quality of the US is poor, the pigeon may look toward the location of the grain, but may not always approach. In that event, the serial compound US is disrupted. When an effect of US duration is reported under these conditions, the effect is suspect, due to confounding by a delay to access or a partial reinforcement outcome (cf. Balsam \& Payne, 1979 , p. 481). In short, the feeder procedure can only be tested under conditions that maintain consistent approach behaviors to the feeder signal. Any change in US strength that weakens the feederapproach sequence results in a breakdown of the compound US.

Because the approach sequence in the feeder procedure is itself a conditioned response, it seems unlikely that a diminution of earlier CRs in the sequence, for example, signal-key pecking, could be obtained before a decline in the feeder-approach sequence occurred. In effect, we are suggesting that the strength of a conditioned response in a serial compound is limited by the strength of the associations later in the sequence, especially when these later associations are instrumental in maintaining the integrity of the compound. Thus, the failure to obtain a clear US-duration effect with the feederduration procedure may not indicate an absence of the effect, but, rather, a limitation on the use of the feeder-duration paradigm. Autoshaping studies have long used the feeder-duration procedure, and its efficacy is well established. However, the serial nature of the feeder US may impose special characteristics. These appear to include a tendency for the strength of the intermediate feeder-approach component to buffer the effect of changing the strength of the final consummatory component.

\section{REFERENCE NOTES}

1. Allison, J. Autoshaping and polydipsia: Leverpressing and drinking as substitutes for eating. Paper presented at the meeting of the Psychonomic Society, Philadelphia, November 1981.

2. Lucas, G. A., \& Deich, J. D. Manuscript in preparation, 1982.

\section{REFERENCES}

Balsam, P. D., Brownstein, A. J., \& Shull, R. L. Effects of varying the duration of grain presentation on automaintenance. Journal of the Experimental Analysis of Behavior, 1978, 29, 27-36.

Balsam, P. D., \& Payne, D. Intertrial interval and unconditioned stimulus durations in autoshaping. Animal Learning \& Behavior, 1979, 7, 477-482.

Bitterman, M. E., Reed, P., \& Krauskopf, J. The effect of the duration of the unconditioned stimulus upon conditioning 
and extinction. American Journal of Psychology, 1952, 65, 256-262.

Brown, P. L., \& Jenkins, H. M. Auto-shaping of the pigeon's key-peck. Journal of the Experimental Analysis of Behavior, 1968, 11, 1-8.

Church, R. M., LoLordo, V., Ovenmier, J. B., Solomon, R. L., \& TURne R, L. H. Cardiac responses to shock in curarized dogs: Effects of shock intensity and duration, warning signal, and prior experience with shock. Journal of Comparative and Physiological Psychology, 1966, 62, 1-7.

Coppock, H. W., \& Chambers, R. M. GSR conditioning: An ilustration of useless distinctions between "types" of conditioning. Psychological Reports, 1959, 5, 171-177.

DEICH, J. D. Second-order conditioning with heat as the primary reinforcer. Unpublished doctoral dissertation, University of lowa, 1982.

Frey, P., \& Butler, C. Rabbit eyelid conditioning as a function of unconditioned stimulus duration. Journal of Comparative and Physiological Psychology, 1973, 85, 289-294.

Furedy, J. J. Classical appetitive conditioning of the GSR with COol air as the UCS, and the roles of the UCS onset and of fset as reinforcers of the CR. Journal of Experimental Psychology, $1967,75,73-80$.

Grbion, J. Scalar expectancy theory and Weber's Law in animal timing. Psychological Review, 1977, 84, 279-325.

GibBon, J. The contingency problem in autoshaping. In C. Locurto, H. S. Terrace, \& J. Gibbon (Eds.), Autoshaping and conditioning theory. New York: Academic Press, 1981.

Gibbon, J., \& BAlsam, P. D. Spreading association in time. In C. Locurto, H. S. Terrace, \& J. Gibbon (Eds.), Autoshaping and conditioning theory. New York: Academic Press, 1981.

Jenkins, H. M., Barnes, R. A., \& Barrera, F. J. Why autoshaping depends on trial spacing. In C. Locurto, H. S. Terrace, \& J. Gibbon (Eds.), Autoshaping and conditioning theory. New York: Academic Press, 1981.

Killeen, P. R. Averaging theory. In C. M. Bradshaw, E. Szabadi, \& C. F. Lowe (Eds.), Quantification of steady-state operant behaviour. Elsevier/North Holland Biomedical Press, 1981.

Locurto, C, Terrace, H. S., \& Gibbon, J. (Eds.). Autoshaping and conditioning theory. New York: Academic Press, 1981.

Mackintos4, N. J. The psychology of animal learning. New York: Academic Press, 1974.

MOWRER, O. H., \& Solomon, L. N. Contiguity vs. drive-reaction in conditioned fear: The proximity and abruptness of drivereduction. American Journal of Psychology, 1954, 67, 15-25.

Overmien, J. B. Instrumental and cardiac indices of Pavlovian fear conditioning as a function of US duration. Journal of Comparative and Physiological Psychology, 1966, 62, 15-20.
Overmer, J. B. Differential Pavlovian fear conditioning as a function of the qualitative nature of the UCS: Constant versus pulsating shock. Conditional Reflex, 1968, 3, 175-180.

RIEss, D., \& FARRAR, C. H. US duration, conditioned acceleration, multiple CR measurement and Pavlovian R-R laws in rats. Journal of Comparative and Physiological Psychology, 1973, 82, 144-151.

Runeuist, W. N., \& Spence, K. W. Performance in eyelid conditioning as a function of UCS duration. Journal of Experimental Psychology, 1959, 57, 249-252.

Salz, E., Kitai, S., \& Asdourian, D. Two-factor theory: Preliminary study of relationship between drive reduction and UCS duration. Psychological Reports, 1963, 12, 757-758.

Snapper, A. G., Stephens, K. R., \& Lee, D. M. The SKED software system. Kalamazoo, Michigan: The SKED Users Group, 1974.

Wasserman, E. A. Pavlovian conditioning with heat reinforcement produces stimulus-directed pecking in chicks. Science, 1973, 181, 875-877.

Wasserman, E. A., Deich, J. D., Hunter, N. B., \& Nagamatsu, L. S. Analyzing the random control procedure: Effects of paired and unpaired CSs and USs on autoshaping the chick's key peck with heat reinforcement. Learning and Motivation, 1977, 8, 467-487.

Wasserman, E. A., Hunter, N. B., Gutowski, K. A., \& BADER, S. A. Autoshaping chicks with heat reinforcement: The role of stimulus-reinforcer and response-reinforcer relations. Journal of Experimental Psychology: Animal Behavior Processes, 1975, 104, 158-169.

Wegner, N., Z Zaman, D. Strength of cardiac conditioned responses with varying unconditioned stimulus durations. Psychological Review, 1958, 65, 238-241.

\section{NOTE}

1. Comparisons between the trial-to-first-peck measure and more stringent acquisition criteria, e.g., trial to fifth peck or trial to begin pecking on three out of four trials (Gibbon \& Balsam, 1981), revealed similar functions. The more lenient criterion was used because the selection of a more demanding criterion would have required excluding additional subjects from the measure.

(Manuscript received February 4, 1982; revision accepted for publication May 3, 1982.) 